\title{
The orbital evolution of satellite galaxies
}

\author{
Yoshikazu Hashimoto and Junichiro Makino \\ Department of Astronomy, School of Science, University of Tokyo \\ Hongo 7-3-1, Bunkyo-ku, Tokyo 113-0033, Japan
}

In most of previous theoretical and numerical works on the orbital evolution of satellite galaxies, the classical Chandrasekhar's formula has been adopted. However, a recent direct N-body result "(van den Bosh et al. 1999)" demonstrated that the orbital evolution is rather different from what is predicted from the Chandrasekhar's formula. The reason for this discrepancy, however, was not clear.

We first verified the result by "van den Bosch et al.". In our simulations the parent galaxy is a King model with $\Psi_{0}=9(\mathrm{~N}=32768)$, and the satellite is a single particle with $1 \%$ mass of parent galaxy (Figure1).

We investigated the reason for the difference between the theoretical work and N-body work. We found that the single largest reason is that in theoretical works the Coulomb logarithm was assumed to be constant throughout the orbit. So, we varied the Coulomb logarithm as,

$$
\ln \Lambda=\frac{1}{2}\left[\ln \left\{1+\ln \left(\frac{b_{\max } V_{s}^{2}}{G(M+m)}\right)^{2}\right\}-\ln \left\{1+\ln \left(\frac{\epsilon_{s} V_{s}^{2}}{G(M+m)}\right)^{2}\right\}\right]
$$

where $b_{\max }=r / 1.4$ and $b_{\min } \simeq \epsilon_{s}$. This improved model with varing the Coulomb logarithm shows very good agreement with the N-body result.

Next, we varied the softening of the satellite galaxy $\epsilon_{s}=0.1,0.0316,0.01$ in N-body simulations and semi-analytical simulations. In both results, the orbit of the satellite whose softening is smaller become circular in earlier stage (Figure2).

For large objects such as satellite galaxies, the assumption that the Coulomb logrithm is constant is invarid. The assumption overestimate dynamical friction around the galactic center and circularization and underestimate the timescale of orbital evolution.

\section{References}

Binney J., Tremaine S., 1987, Galactic Dynamics. Princeton Univ. Press, Princeton

Frank C., van den Bosch, G. F. L., G. L., J. S. , 1999, ApJ, 515, 50

Ing-Guey Jiang, J. Binney, 2000, MNRAS, 314, 468 

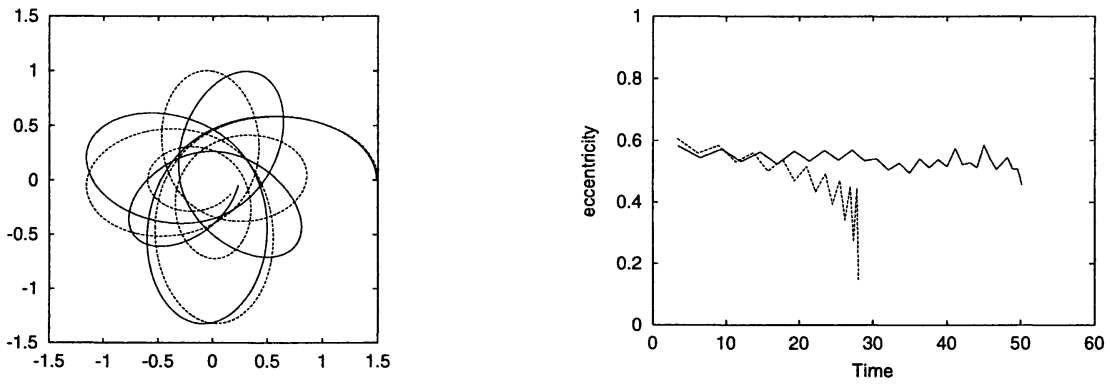

Figure 1. The left panel shows the orbits of satellites, and the right panel shows the orbital eccentricities of the satellites as a function of time. In both panels, full curve shows $\mathrm{N}$-body simulation and dashed curve is that of semi-analytical simulation.
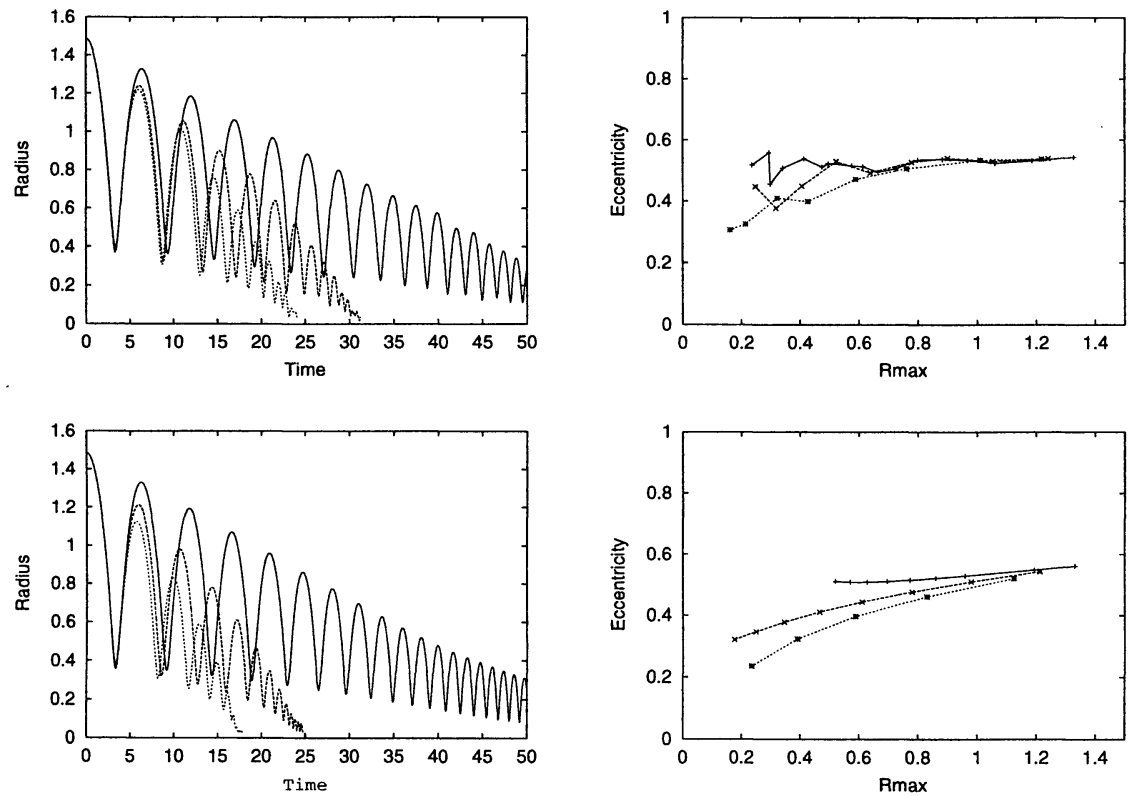

Figure 2. The upper panels are results of N-body simulations, and the lower panels are results of semi-analytical simulations with the modefied dynamical friction fomula. The left panels shows distance of the satellites from the galactic center versus time, and the right panels shows orbital ecentricity versus apocentric distance. Full, dashed, and dotted curves represent the results with $\epsilon_{s}=0.1,0.0316,0.01$, respectively. 